Departamento de Farmacia, Facultad de Farmacia, Universidad de Concepción. Concepción, Chile.

${ }^{a} Q u i ́ m i c o$ Farmacéutico, Magíster en Ciencias Farmacéuticas, Doctor en Ciencias Biológicas, área Botánica.

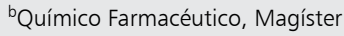
en Salud Familiar.

'Químico Farmacéutico, Magíster en Ciencias Farmacéuticas, Doctor en Farmacología.

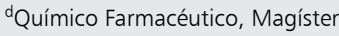
en Ciencias Farmacéuticas.

Recibido el 22 de octubre de 2015, aceptado el 26 de agosto de 2016.

Correspondencia a:

Dra. Marcia Avello L.

Departamento de Farmacia, Facultad de Farmacia, Universidad de Concepción. Barrio Universitario $s / \mathrm{n}$. maavello@udec.cl

\section{Potencial uso terapéutico de cannabis}

\author{
MARCIA AVELLO L. ${ }^{a}$, EDGAR PASTENE N. ${ }^{\text {, }}$ \\ POLA FERNÁNDEZ R. ${ }^{c}$, PIA CÓRDOVA M. ${ }^{d}$
}

\section{Therapeutic potential of Cannabis sativa}

Cannabis sativa (marihuana) is considered an illicit drug due to its psychoactive properties. Recently, the Chilean government opened to the use cannabis in the symptomatic treatment of some patients. The biological effects of cannabis render it useful for the complementary treatment of specific clinical situations such as chronic pain. We retrieved scientific information about the analgesic properties of cannabis, using it as a safe drug. The drug may block or inhibit the transmission of nervous impulses at different levels, an effect associated with pain control. Within this context and using adequate doses, forms and administration pathways, it can be used for chronic pain management, considering its effectiveness and low cost. It could also be considered as an alternative in patients receiving prolonged analgesic therapies with multiple adverse effects.

(Rev Med Chile 2017; 145: 360-367)

Key words: Cannabis Sativa; Chronic Pain; Therapeutics.
( annabis sativa es una angiosperma originaria de Asia, tiene una amplia distribución debido a su adaptación geoclimática y ecosistémica ${ }^{1}$.

La etnobotánica describe su uso medicinal, en rituales religiosos y ha sido importante materia prima para el sustento de la industria del papel, las telas y la construcción ${ }^{1}$. Cannabis fue desplazada lentamente como recurso medicinal, hasta que en los años 1950-1960 se la eliminó de estos libros académicos completamente, y se la catalogó como droga de uso ilícito por su potencial efecto sicoactivo y de abuso, asociándola a otras drogas de uso ilícito ${ }^{1}$.

Según el concepto de droga de uso ilícito, se la clasifica de acuerdo al contenido en THC $\left(\Delta^{9} \text {-tetrahidrocannabinol) (Tabla } 1\right)^{1}$.

El contenido de estos marcadores químicos se asocia con la región geoclimática de origen ${ }^{1}$. En Chile (clima mediterráneo), se encuentra el tipo "intermedio". Este tipo y el "de droga" son los más perseguidos por constituir las materias primas más requeridas para el uso ilícito. En Chile, el uso lúdico y recreativo de cannabis es ilegal y se ampara en la Ley 20.000. Según el "XI Estudio Nacional de Drogas en Población General de Chile, 2014”, del SENDA (Servicio Nacional para la Prevención y Rehabilitación del Consumo de Drogas y Alcohol)el consumo de cannabis marca una tendencia ascendente, (reportes año $2010 \mathrm{al} 2014$ ) donde se observa que de una prevalencia de consumo de un $23,0 \%$ en 2012, se llegó a 31,5\% en 2014. Por su parte, las declaraciones de consumo de marihuana en adolescentes de 12 a 18 años presentaron un alza significativa respecto de 2012 , variando de $6,7 \%$ en 2012 a 13,5\% en 2014. En esta población el uso de cannabis se considera injuriante para el desarrollo del sistema nervioso central.

Como recurso medicinal cannabis es una "planta de actividad intermedia" 2 . Tiene un margen terapéutico relativamente amplio, pero con efectos adversos que deben ser monitoreados ${ }^{3}$, por lo que estaría cumpliendo los requisitos según los antecedentes compilados hasta ahora, como tratamiento complementario de trastornos crónicos ${ }^{2}$.

\section{Cannabis y Cannabinoides}

Cannabis es una mezcla compleja de principios activos y acompañantes que favorecen la biodisponibilidad de los activos, modulan la sinergia y atenúan efectos tóxicos. Se han identificado 400 
Tabla 1. Clasificación de cannabis según contenido de THC ( $\Delta^{9}$-tetrahidrocannabinol)

\begin{tabular}{|ll|}
\hline Clasificación & Contenido de THC (\%) \\
Droga & $>1 \%$ \\
Fibra & $<0,3 \%$ \\
Intermedio & $\begin{array}{l}\text { THC y CBD, cannabidiol, en proporcio- } \\
\text { nes similares }\end{array}$ \\
\hline
\end{tabular}

tipos de principios activos de familias fitoquímicas diferentes. De éstos, 60 pertenecen al grupo de los cannabinoides $^{1,2}$.

El ser humano presenta una serie de receptores de membrana multifuncionales que responden a moléculas que, sin ser cannabinoides, se comportan como tales, son los llamados endocannabinoides ${ }^{4}$. Los endocannabinoides son lípidos bioactivos que al interaccionar con estas proteínas de membrana, causan efectos biológicos similares a los cannabinoides pero fisiológicamente controlados. Estas proteínas son los receptores CB1 y CB2, los cuales forman parte de la familia de receptores de 7 transmembrana, acoplados a proteínas G inhibitorias (GPCRs). El receptor CB1 es un polipéptidodo de 472 aminoácidos, siendo el más abundante y de mayor distribución en el organismo humano. Se concentra en el cerebro, médula espinal y regiones sensoriales periféricas, donde modula funciones cerebrales como aprendizaje, memoria, emociones, movimiento, ciclos circadianos y de regulación central, además de funciones endocrinas, metabólicas y de balance energético ${ }^{4-6}$.

El receptor CB2 es un polipéptidodo de 342 aminoácidos, de menor abundancia y distribución, localizado en tejidos periféricos. Se le responsabiliza de la modulación de procesos inflamatorios e inmunitarios, por lo que es objeto de estudio por su potencial intervención en procesos tumorales?.

Un aspecto interesante del receptor $\mathrm{CB} 1$ es que interacciona con otros receptores como los dopaminérgicos, opiodes, valínicos y de orexina- $1^{8}$. El THC se une con similar afinidad a receptores CB1 y CB2. Aunque los canabinoides actúan en receptores $\mathrm{CB}$, ciertos efectos, como los asociados al control de las náuseas y el vómito, están relacionados con su interacción con receptores de serotonina 5-HT3, donde actúan como antagonistas ${ }^{8}$.
$\mathrm{Al}$ respecto, hace más de una década que en Chile se retomó la discusión de la posible contribución medicinal de cannabis.

\section{Cannabis medicinal en Chile}

El año 2014, el gobierno de Chile dio indicios de responder ante la necesidad de ofrecer soluciones médicas a pacientes que requieren el apoyo sintomático de cannabis. Esta iniciativa busca hacer menos engorrosos los trámites de los pacientes en cuanto a su consumo, puesto que el uso terapéutico está contemplado en la Ley 20.000.

Hoy día el gobierno de Chile trabaja hacia una legislación con carácter nacional para atender el problema de acceso a cannabis medicinal con estándares claros para determinar casos, formas de prescripción, y acceso a derivados de cannabis.

La opción inmediata para utilizar este recurso natural con fines terapéuticos en Chile, es como planta en su estado natural, o un producto obtenido directamente de sus partes botánicas como son los extractos crudos. Hoy, en nuestro medio, estos principios activos serían muy costosos pensando en la importación, puesto que no se ha determinado hasta ahora una estrategia farmacéutica para obtenerlos en nuestro país.

Uno de los puntos críticos para la utilización segura de plantas medicinales en su estado natural o sus extractos, tiene que ver con la estandarización de su cultivo y el consecuente contenido en principios activos según las características geoclimáticas del terreno donde crezcan ${ }^{1}$.

Cannabis es una planta que se adapta a muchos ecosistemas, y lo hace manipulando las rutas biosintéticas de compuestos fitoquímicos que le permiten sobrevivir y ser exitosa en territorios muy diferentes entre síl. Es por esto que los especímenes que crecen en diferentes latitudes, a pesar de ser una misma especie, no son comparables químicamente. Así, es necesario conocer exactamente las características químicas de las plantas que crecen en nuestro territorio para poder establecer una dosis efectiva y segura.

\section{Aplicaciones}

Investigadores de todo el mundo disputan fuertemente acerca del uso medicinal de cannabis $^{9-20}$. 
Tabla 2. Algunas aplicaciones terapéuticas de cannabis y sus derivados

\begin{tabular}{|ll|}
\hline Categoría terapéutica & Potenciación cannabinoide \\
\hline A & Analgésico \\
Tratamiento complementario & Trastornos espásticos \\
& Náuseas y vómitos inducidos por quimioterapia \\
\hline B & Síndrome caquéctico-anoréxico en pacientes con SIDA o cáncer terminal \\
No se consideran tratamientos complementarios & Glaucoma \\
por existir otros mejores & Trastornos inflamatorios del tubo digestivo \\
& Ansiedad, depresión \\
& Patología tumoral \\
& Neuroprotección \\
\hline
\end{tabular}

Las aplicaciones terapéuticas para cannabis y sus derivados son muy amplias; se describen acciones agonistas y antagonistas cannabinoides. Por las manifestaciones adversas y efectos sicoactivos, los agonistas cannabinoides se han descrito como tratamiento complementario para algunas patologías (Tabla 2A). Para otras (Tabla 2B), existen mejores tratamientos o son campos nuevos de investigación ${ }^{21}$. Mientras que para los antagonistas cannabinoides, cuyo representante natural es el CBD, se han propuesto ciertas acciones como tratamiento para la obesidad, dislipidemias y adicciones, entre otras ${ }^{22,23}$.

Para evitar efectos adversos y sicoactivos se están diseñando análogos sintéticos que permitan mejorar la efectividad terapéutica. Se propone el uso de agonistas CB1 que no crucen la barrera hemato encefálica, agonistas selectivos CB2, manipular el sistema endocannabinoide minimizando la aparición de efectos adversos, o desarrollar nuevas vías de administración ${ }^{24}$.

No todos los cannabinoides comparten las mismas características químicas. Si bien son productos de una misma ruta metabólica tienen grupos funcionales y cadenas carbonadas que los hacen diferentes. Es así como los cannabinoides tipo THC, son más lipofílicos, tienen una acción central importante y son los principales fármacos que actúan sobre el receptor CB1. Mientras que otros como el CBD, se encuentran en forma de derivados carboxílicos, acompañados de sus homólogos de cadena lateral más corta lo que les confiere menos lipofilicidad y más acción periférica. En respuesta a estas características y afinidad por los receptores CBs, se han descrito diversos efectos biológicos en blancos específicos ${ }^{25-27}$.

Uno de los aspectos controversiales de can- nabis tiene que ver con su efecto negativo sobre el aprendizaje, particularmente en individuos jóvenes expuestos a ella. En un estudio reciente ${ }^{31}$, se evaluó el efecto del consumo de marihuana en 28 adultos jóvenes, los cuales fueron estratificados en consumidores tempranos y consumidores tardíos. En los individuos con consumo temprano se observó mayor lentitud en el aprendizaje de palabras, pero no se observaron efectos amnésicos. Interesantemente, el número de palabras aprendidas fue similar entre el grupo control y el de inicio tardío. Este mismo año en otro estudio ${ }^{32}$ en primates no-humanos, en los que evaluaron el efecto del THC, anandamida, URB597 (inhibidor de la amido hidrolasa de ácidos grasos, FAAH) y metanandamida (análogo metabólicamente estable de anandamida), sobre el aprendizaje, flexibilidad cognitiva, memoria a corto plazo, atención, y motivación. Ellos observaron alteraciones cognitivas las que, en orden de importancia se reflejaron en una disminución de la capacidad discriminativa, aprendizaje, flexibilidad y memoria a corto plazo. No obstante, dichos efectos no se observaron para la anandamida y URB597. Además, los derivados metabólicamente estables no afectaron la atención, lo que sugiere que tendrían ventajas terapéuticas para su futuro uso clínico.

Uno de los efectos más apreciados de cannabis en terapia, según lo mostrado en la Tabla 2A, sería el efecto analgésico ${ }^{21,28-30}$.

\section{Cannabis y dolor}

Cannabis y sus derivados son capaces de bloquear o inhibir la transmisión del impulso nervioso a varios niveles. Esta actividad farma- 
cológica parece ejercerse a través de la activación de receptores $\mathrm{CB} 1$ a nivel del cerebro, la médula espinal, y las neuronas sensoriales periféricas. Se describe que los receptores cannabinoides están acoplados a proteínas $\mathrm{G}$ inhibidoras, que inhiben la activación de la adenilatociclasa y la entrada de calcio al interior celular y favorecen la salida de potasio. Como consecuencia, disminuyen la excitabilidad de la membrana y la actividad neuronal, por lo tanto, el resultado final es la reducción de la liberación de neurotransmisores ${ }^{33}$.

Cannabis y sus derivados estarían asociados al control de dolor crónico derivado de procesos cancerosos y el dolor neuropático ${ }^{21,33}$.

En el dolor canceroso es habitual la utilización de opiáceos, sin embargo, su uso continuo supone la aparición de tolerancia. Dado que opiáceos y cannabinoides producen un efecto analgésico a través de un mecanismo similar, que bloquea tanto en el cerebro, como en la médula espinal la liberación de neurotransmisores implicados en la transmisión del dolor, es que se propone que los cannabinoides aumentan la potencia analgésica de diversos opiáceos. De esta forma existe la posibilidad de combinarlos buscando potenciación del efecto analgésico y menor riesgo de aparición de efectos secundarios, tanto de opiáceos como de cannabinoides, apoyado también en que se ha observado que para los cannabinoides se desarrolla tolerancia más rápidamente a los efectos adversos que a los terapéuticos ${ }^{13,20}$.

Se han publicado varios ensayos clínicos que examinan el uso de los agonistas del receptor de cannabinoides para aliviar el dolor del cáncer crónico. Sin embargo, los estudios están limitados por el tamaño pequeño de muestra y el hecho de que todos los pacientes también recibieron su tratamiento analgésico habitual junto con un preparado de THC o un placebo. Además los pacientes señalan presentar sedación a las dosis de analgésicas de THC, lo cual puede limitar su uso $^{34}$. Abrams, 2011, realizó un estudio en que se administró cannabis a la forma vaporizada junto con morfina de liberación sostenida u oxicodona durante cinco días, concluyendo que la administración de cannabis a la forma vaporizada aumentaba el efecto analgésico de los opioides, sin alterar significativamente sus niveles plasmáticos, lo que permitiría reducir las dosis de éstos y disminuir los efectos adversos ${ }^{35}$.

Los resultados sugieren un beneficio como complemento a la terapia tradicional, pero se necesitan más ensayos clínicos para evaluar los efectos del cannabis y sus otros derivados ${ }^{34}$.

El dolor neuropático supone una manifestación de un trastorno en las vías de transmisión sensorial del dolor. Cannabis y sus derivados han mostrado ser altamente efectivos en el dolor neuropático debido a que actuarían principalmente inhibiendo o liberando una serie de moduladores desde las neuronas y/o tejidos no neuronales. Es por esto que su efecto analgésico sería más rápido e intenso en tejidos lesionados o inflamados, donde dichos moduladores, sustancias proinflamatorias y proalgésicas, se encontrarían en cantidades mucho mayores, haciendo que la transmisión del estímulo doloroso sea directamente dependiente a su liberación. Estas sustancias que mantienen y potencian la transmisión dolorosa, son responsables de la cronificación y amplificación del dolor ${ }^{36}$. Al respecto, se evalúa la planta a la forma de cigarrillo o vaporizada comparado con un placebo, observándose un prometedor efecto analgésico, principalmente en aquellos preparados con mayor concentración de THC (9,6\%). El problema de estos estudios, es que incluyen una pequeña muestra de pacientes y los cigarrillos empleados contenían distintas concentraciones de $\mathrm{THC}^{37-40}$.

En la Tabla 2A, se establece como principales aplicaciones terapéuticas de cannabis y sus derivados, como agonistas cannabinoides, los efectos analgésico y antiespástico. El dolor refractario que caracteriza la esclerosis múltiple, es de naturaleza espástica. En diferentes modelos de esclerosis múltiple se ha observado que la espasticidad estaría controlada por el sistema cannabinoide endógeno. En este sentido, la activación de los receptores CB1 por parte de cannabis y sus derivados podría tener un efecto beneficioso en la inhibición de la espasticidad. La sinergia analgésica dada por la inhibición de la transmisión del impulso nervioso y el efecto antiespástico que involucra a cannabis y sus derivados en este cuadro, permite que el efecto analgésico neto sea muy efectivo ${ }^{29,37,41,43-45}$. Sin embargo, otros autores presentan resultados contradictorios. En un ensayo clínico aleatorizado, doble ciego, multicéntrico, con grupos paralelos y controlado con placebo, se estudió la efectividad y seguridad del uso de THC en la esclerosis múltiple progresiva en 27 centros del Reino Unido. Los voluntarios fueron adultos entre 18-65 años con esclerosis múltiple primaria y secundaria y 
con un año de progresión de la enfermedad y una puntuación basal de 4,0-6,5 en la Escala Expandida de Estatus de Discapacidad. La intervención de 28 $\mathrm{mg} /$ día con THC o placebo no fue suficiente para promover efectos significativamente diferentes entre ambos grupos. Si bien no se observaron problemas de seguridad, algunos efectos indeseados afectaron el cumplimento del tratamiento. Los autores destacan que el tratamiento tampoco resulto en una relación costo-efectividad favorable.

Las limitaciones que presentan la mayoría de los estudios que evalúan el efecto analgésico de cannabis son las diferentes dosis y formas farmacéuticas utilizadas, la falta de ensayos comparativos para las distintas formulaciones y vías de administración, el reducido tamaño de muestra, y el sesgo de la selección de pacientes ${ }^{37}$.

Autores ${ }^{21,28-38}$ proponen el apoyo de cannabis y sus derivados en los tipos de dolores mencionados, como el canceroso, neuropático y espástico (Tabla 3), siendo la principal enfermedad beneficiaria de la analgesia producida por cannabis la esclerosis múltiple y enfermedades que cursen con dolor neuropático. Este apoyo se propone en cualquier estadio del dolor, y como complemento a analgésicos típicos para cada una de estas enfermedades.

\section{Cannabis, recurso terapéutico inmediato}

Como recurso terapéutico inmediato para Chile, cannabis podría ser usada como planta en su estado natural. Esto implica la acción conjunta de los principios activos de plantas que crecen en un determinado territorio con condiciones geoclimáticas características. Por lo tanto, la concentración de THC y CBD, marcadores por excelencia de cannabis, va a cambiar en función de esto.

Como muestra la Tabla 3 para las patologías expuestas, se utiliza cannabis a la forma de extracto o inhalada, formas de uso de la planta en su estado natural. El extracto o aceite (vía oral) es un producto concentrado representante de la fitoquímica de la planta que tiene afinidad con un solvente determinado. En los casos expuestos en la Tabla 3 , se trata de un extracto estandarizado en una relación THC:CBD (1:1), correspondientes a 2,7:2,5 $\mathrm{mg}$, respectivamente. La importancia de conocer la composición química de productos como este permite dosificar, y a su vez hacer farmacología ${ }^{46}$.

Las inhalaciones en cambio, dificultan la exacta

\section{Tabla 3. Patologías que cursan con dolor y derivados de cannabis como tratamiento complementario}

\begin{tabular}{|ll|}
\hline Patologías & Presentaciones \\
\hline Esclerosis múltiple & $\begin{array}{l}\text { Extractos } \\
\text { Cannabis inhalada } \\
\text { Dronabinol (THC) } \\
\text { Nabilona (análogo sintético THC) } \\
\text { THC+CBD }\end{array}$ \\
$\begin{array}{ll}\text { Dolor neuropático en } \\
\text { pacientes con VIH }\end{array}$ & Extractos \\
Dolor neuropático en & Extractos inhalada \\
diferentes patologías & Cannabis inhalada \\
& Nabilona \\
& THC+CBD \\
\hline
\end{tabular}

dosificación, por la naturaleza de vapor a la que queda reducida la muestra ${ }^{47}$.

La vía de administración más efectiva es la inhalación. Pese al elevado coeficiente de partición de los canabinoles, la vía oral es errática y poco efectiva por las barreras naturales y la degradación gastrointestinal que sufren los principios activos antes de su absorción. La distribución de los cannabinoides, por su naturaleza lipofílica, es amplia y su liberación lenta. Tienen un metabolismo hepático, pulmonar e intestinal rápido, dependiendo de la vía de administración. Son de eliminación renal y mayoritariamente fecal. La vida media de los metabolitos varía entre 3 a 20 días, dependiendo del metabolito buscado. En el plasma es posible detectar los metabolitos 11-O-THC y THC-COOH, los cuales aparecen en menor concentración que el THC en las primeras horas, pero pueden alcanzar niveles mayores y circular en el plasma más de 20 h después de dos dosis oral repetidas de THC. Respecto de lo anterior, se ha logrado mayor éxito en alcanzar concentraciones terapéuticamente efectivas usando la vía sublingual para el manejo del dolor neuropático y los síntomas de la esclerosis múltiple ${ }^{47-51}$.

Los efectos adversos del uso de cannabinoides en el tratamiento del dolor se clasifican en centrales y periféricos. Centrales como mareos, cansancio y/o debilidad, somnolencia y alteraciones de la memoria, y periféricos como sequedad en la boca, náuseas, hipotensión transitoria, y mialgias o debilidad muscular. En ningún caso se describen efectos graves y siempre son reversibles 
tras la suspensión del tratamiento. El desarrollo de estos efectos indeseables, así como del efecto analgésico va a depender de la susceptibilidad del paciente. Así, hoy se recomienda ajustar la dosis y vía de administración en forma empírica según la respuesta del paciente ${ }^{52}$.

Con respecto alas precauciones, se describen mayoritariamente contraindicaciones en pacientes que estén cursando con enfermedades importantes sicopáticas, cardíacas y con condiciones delicadas, como en embarazo, lactancia y adultos mayores con polifarmacia. Las interacciones medicamentosas derivan de las contraindicaciones ya descritas ${ }^{52}$.

También se describe ausencia de tolerancia. Con respecto al síndrome de abstinencia, los dos primeros días son de cuidado, y se necesita de hasta dos semanas para la desaparición total de los síntomas, siendo éstos mejor llevados que para otras drogas ${ }^{53}$.

Se recomienda, para los clínicos en momentos donde no hay desarrollo de farmacología para cannabis, aumentar la dosis poco a poco esperando encontrar el efecto deseado, para cada paciente según la forma de usos populares ${ }^{52}$.

Para poder hacer un uso más efectivo y seguro se propone desarrollar nuevas estrategias terapéuticas para disminuir efectos adversos y aumentar la efectividad, así como estandarizar la planta.

Se aconseja a futuro, que las investigaciones para evaluar el uso terapéutico de cannabis, incluyan formulaciones y/o dosis estándar en poblaciones de estudio más grandes. También es importante incorporar en los preparados con THC la medición de CDB para determinar si los efectos no psicotrópicos de este compuesto pueden mejorar la tolerancia y la seguridad de THC. Lo importante es determinar el uso seguro y eficaz de cannabis ${ }^{54}$.

\section{Referencias}

1. Fassio A, Rodríguez M, Ceretta S. Unidad de Comunicación y Transferencia de Tecnología de INIA, Editores, Cáñamo (Cannabis sativa L.). Uruguay; 2013. 96.

2. Amar BA. Cannabinoids in medicine: A review of their therapeutic potential. J Ethnopharmacol 2006; 105: 1-25.

3. Avello M, Cisternas I. Fitoterapia, sus orígenes, características, y situación en Chile. Rev Med Chile 2010; 138: 1278-83.

4. Bisogno T. Endogenous cannabinoids: Structure and metabolism. J Neuroendocrinol 2008; 20: 1-9.
5. Bellocchino L, Cervino C, Pasquali R, Pagotto U. Theendo cannabinoid system and energy metabolism. J Neuroendocrinol 2008; 20: 850-7.

6. Battista N, Rapino C, di Tommaso M, Bari M, Pasquariello N, Maccarrone $\mathrm{M}$. Regulation of male fertility by theen docannabinoid system. Mol Cell Endocrinol 2008; 286S: S17-23.

7. Brown AJ. Novel cannabinoid receptors. Br J Pharmacol 2007; 152: 567-75.

8. Ferre'S, Baler R, Bouvier M, Caron MG, Devi LA, Durroux T, et al. Building a new conceptual frame work for receptor heteromers. Nat Chem Biol 2009a; 5: 131-4.

9. Pertwee RG. The diverse $\mathrm{CB} 1$ and $\mathrm{CB} 2$ receptor pharmacology of three plant cannabinoids: delta9-tetrahydrocannabinol, cannabidiol and delta9-tetrahydrocannabivarin. Br J Pharmacol 2008; 153: 199-215.

10. Bostwick JM. Blurred boundaries: the therapeutics and politics of medical marijuana. Mayo Clin Proc 2012; 87: 172-86.

11. Mechoulam R. Cannabis-a valuable drug that deserves better treatment. Mayo Clin Proc 2012; 87: 107-9.

12. Nussbaum AM, Boyer JA, Kondrad EC. "But my doctor recommended pot": medical marijuana and the patient-physician relationship. J Gen Med 2011; 26: 1364-7.

13. Bowles DW, O'Bryant CL, Camidge DR, Jimeno A. The intersection between cannabis and cancer in the United States. Crit Rev Oncol Hematol 2012; 83: 1-10.

14. Kleber JD, DuPont RL. Physicians and medical marijuana. Am J Psychiatry 2012; 169: 564-8.

15. McPartland JM, Pruitt PL. Medical marijuana and its use by the immunocompromised. Altern Ther Health Med 1997; 3: 39-45.

16. Battistella G, Fornari E, Thomas A, Mall JF, Chtioui H, Appenzeller M, et al. Weed or wheel! FMRI, behavioural, and toxicological investigations of how cannabis smoking affects skills necessary for driving. PLoS One 2013; 8(1): e52545.

17. Henstridge CM. Off-target cannabinoid effects mediated by GPR55. Pharmacology 2012; 89: 179-87.

18. Sharir H, Abood ME. Pharmacological characterization of GPR55, aputative cannabinoid receptor. Pharmacol Ther 2010; 126: 301-13.

19. Moreira FA, Grieb M, Lutz B. Central side-effects of therapies based on CB1 cannabinoid receptor agonists and antagonists: focus on anxiety and depression. Best Pract Res Clin Endocrinol Metab 2009; 23: 133-44.

20. Irvin W Jr, Muss HB, Mayer DK. Symptom management in metastatic breast cancer. Oncologist 2011; 16: 1203-14. 
21. Manzanares J, Julian M, Carrascosa A. Role of the cannabinoid system in pain control and therapeutic implications for the management of acute and chronic pain episodes. Curr Neuropharmacol 2006; 4: 239-57.

22. Callado LF, Meana JJ, Grau L, Gonzalvo B. Cannabis. En: Bobes J, Casa M, Gutiérrez M, Editores, Manual de Trastornos Adictivos. Madrid, España: Enfoque Editorial; 2011. p. 440-7.

23. Donoghue K, Doody GA, Murray RM, Jones PB, Morgan C, Dazzan P, et al. Cannabis use, gender and age of onset of schizophrenia: data fromthe AESOP study. Psychiatry Res 2014; 215: 528-32.

24. Kapur B, Lala P, Shaw J. Pharmacogenetics of chronic pain management. Clin Biochem 2014; 47: 1169-87.

25. Pertwee RG. Emerging strategies for exploiting cannabinoid receptor agonists as medicines. Brit J Pharmacol 2009; 156: 397-411.

26. Callado LF. Utilización terapéutica de Cannabis. En Arana X, Markez I, Editores, Cannabis: Salud, Legislación y Políticas de Intervención. Madrid, España: Editorial Dykinson; 2006. p. 73-90.

27. Callado LF, Pomposo I, Garibi J. Rimonabant. En: Salazar M, Peralta C, Pastor FJ, Editores, Tratado de Psicofarmacología: Bases y Aplicación Clínica. Madrid, España: Editorial Médica Panamericana; 2009. p. 41921.

28. Abrams DI, Jay CA, Shade SB, Vizoso H, Reda H, Press $S$, et al. Cannabis in painful HIV-associated sensory neuropathy: a randomized placebo-controlled trial. Neurology 2007; 68: 515-21.

29. Rog DJ, Nurmikko TJ, Friede T, Young CA. Randomized, controlled trial of cannabis-based medicine in central pain in multiplesclerosis. Neurology 2005; 65: 812-9.

30. Ware M, Beaulieu P. Cannabinoids for the treatment of pain: an update onrecent clinical trials. Pain Res Manag 2005; 10: 27a-30a.

31. Schuster R, Hoeppner SS, Evins AE, Gilman JM. Early onset marijuana use is associated with learning inefficiencies. Neuropsychology 2016; 30(4): 404-15.

32. Kangas BD, Leonard MZ, Shukla VG, Alapafuja SO, Nikas SP, Makriyannis A, et al. Comparisons of D9-tetrahydrocannabinol and anandamide on a battery of cognition-related behavior in nonhuman primates. J Pharmacol Exp Ther 2016; 357 (1): 125-33.

33. Clark AJ, Lynch ME, Ware M, Beaulieu P, McGilveray IJ, Gourlay D. Guidelines for the use of cannabinoid compounds in chronic pain. Pain Res Manag 2005; 10: $44 a-6 a$.

34. Wilkie G, Sakr B, Rizack T. Medical marijuana use in oncology: A review. JAMA Oncol 2016; 2 (5): 670-5.
35. Abrams DI, Couey P, Shade SB, Kelly ME, Benowitz NL. Cannabinoid-opioid interaction in chronic pain. Clin Pharmacol Ther 2011; 90 (6): 844-51.

36. Ellis RJ, Toperoff W, Vaida F, van den BG, Gonzales J, Gouaux B, et al. Smoked medicinal cannabis for neuropathic pain in HIV: a randomized, crossover clinical trial. Neuropsychopharmacology 2009; 34: 672-80.

37. Ware MA, Wang T, Shapiro S, Robinson A, Ducruet T, Huynh T, et al. Smoked cannabis for chronic neuropathic pain: a randomized controlled trial. CMAJ 2010; 182: E694-701.

38. Wallace 1, Schulteis G, Atkinson JH, Wolfson T, Lazzaretto D, Bentley H, et al. Dose-dependent effects of smoked cannabis on capsaicin-induced pain and hyperalgesia in healthy volunteers. Anesthesiology 2007; 107: 785-96.

39. Wilsey B, Marcotte T, Tsodikov A, Millman J, Bentley $\mathrm{H}$, Gouaux B, et al. A randomized, placebo-controlled, crossover trial of cannabis cigarettes. J Pain 2008; 9 (6): 506-21.

40.- Andreae MH, Carter GM, Shaparin N, Suslov K, Ellis RJ, Ware MA, et al. Inhaled cannabis for chronic neuropathic pain: A meta-analysis of individual patient data. J Pain 2015; 16 (12): 1221-32.

41. Borgelt LM, Franson KL, Nussbaum AM, Wang GS. The pharmacologic and clinical effects of medical cannabis. Pharmacotherapy 2013; 33 (2): 195-209.

42. Naftali T, Schleider LRL, Dotan I, Lansky EP, Benjaminov FS, Konikoff FM. Cannabis induces a clinical response in patients with Crohn's disease: a prospective placebo-controlled study. Clin Gastroenterol Hepatol 2013; 11: 1276-80.

43. Koppel BS, Brust JC, Fife T, Bronstein J, Youssof S, Gronseth G, et al. Systematic review: Efficacy and safety of medical marijuana in selected neurologic disorders: Report of the guideline development subcommittee of the American Academy of Neurology. Neurology 2014; 82 (17): 1556-63.

44. Novotna A, Mares J, Ratcliffe S, Novakova I, Vachova $\mathrm{M}$, Zapletalova $\mathrm{O}$, et al. A randomized double-blind-placebo-controlled, parallel-group, enriched-design study of nabiximols ${ }^{*}$ (Sativex ${ }^{\circledR}$ ), as add-on therapy, in subjects with refractory spasticity caused by multiple sclerosis. Eur J Neurol 2011; 18 (9): 1122-31.

45. Pavisian B, MacIntosh BJ, Szilagyi G, Staines RW, O'Connor P, Feinstein A. Effects of cannabis on cognition in patients with MS: a psychometric and MRI study. Neurology 2014; 82 (21): 1879-87.

46. Savage SR, Romero-Sandoval A, Schatman M, Wallace M, Fanciullo G, McCarberg B, et al. Cannabis in pain treatment: clinical and research considerations. J Pain 2016; 17 (6): 654-68. 
47. Zajicek JP, Sanders HP, Wright DE, Vickery PJ, Ingram WM, Reilly SM, et al. Cannabinoids in multiplesclerosis (cams) study: safety and efficacy data for 12 months follow up. J Neurol Neurosurg Psychiatry 2005; 76: 1664-9.

48. Leung L. Cannabis and anditsderivatives: review of medical use. J Am Board Fam Med 2011; 24: 452-62.

49. Vaney C, Heinzel-Gutenbrunner M, Jobin P, Tschopp F, Gattlen B, Hagen U, et al. Efficacy, safety and tolerability of an orally administered cannabis extract in thetreatment of spasticity in patients with multiplesclerosis: a randomized, double-blind, placebo-controlled, crossover study. Mult Scler 2004; 10: 417-24.

50. Walker JM, Huang SM. Cannabinoid analgesia. Pharmacol Ther 2002; 95: 127-35.

51. Nadulski T, Sporkert F, Schnelle M, Stadelmann AM, Roser P, Schefter T, et al. Simultaneous and sensitive analysis of THC, 11-OH-THC, THC-COOH, CBD, and CBN by GC-MS in plasma after oral application of small doses of THC and cannabis extract. J Anal Toxicol 2005; 29: 782-9.

52. Vaughan CW, Christie MJ. Cannabis and Cannabinoids: Pharmacology, Toxicology, and Therapeutic Potential. Grotenhermen F, Russo E, Editores. New York: The Haworth Integrative Healing Press; 1984. p. 89.

53. Holdcroft A. Cannabis and Cannabinoids: Pharmacology, Toxicology, and Therapeutic Potential. Grotenhermen F, Russo E, Editores. New York: The Haworth Integrative Healing Press; 1984. p. 181.

54. Zajicek J, Fox P, Sanders H, Wright D, Vickery J, Nunn A, et al. Cannabinoids for treatment of spasticity and other symptoms related to multiple sclerosis (CAMS study): multicenter randomised placebo-controlled trial. Lancet 2003; 362: 1517-26. 